\title{
Infrared thermospectroscopic 2D and 3D imaging of confined drying process
}

\author{
by M. Lehtihet *, J. Leng * and C. Pradère** \\ * Laboratory of the Future, CNRS/Solvay UMR no 5258, 178 avenue du Dr Albert Schweitzer, 33608 Pessac, \\ France \\ ${ }^{* *}$ I2M TREFLE, UMR CNRS no 5295, Esplanade des Arts et Metiers, 33405 Talence, France
}

\begin{abstract}
In this work is presented an InfraRed (IR) imaging technique allowing one to retrieve quantitative concentration and temperature maps with relatively fast acquisition times [1]. Dynamic samples can be imaged with micrometric spatial resolution and a complete study of coupled heat and mass transfer phenomena can be realized on the basis of these images. A proof-of-concept of this technique is realized on a model transient problem : the drying of a $\mu \mathrm{L}$ drop of colloidal dispersion in confined geometry [2]. Quantitative maps of concentration and temperature everywhere in the drop inside the cell of the thermospectrometer are retrieved. Transport phenomena like colloids re-distribution inside the droplet due to inhomoegeneous drying can be highlighted by this mean. A numerical inverse method based on the acquired images is also established, allowing one to estimate intrinsic properties of the studied material as the diffusivity coefficient [3]. Also, thermal images of the same drying process are presented and temperature gradients establishing themselves due to the vaporization enthalpy are measured and validated using a thermal model of the confined drop. Finally, to extend the setup sensitivity to the thickness of the sample, an IR tomography technique inspired of confocal microscopy is applied on the ending result of a confined dying of silica dispersion : a millimetric silica gel. By this method, tomographs of both spectroscopic and thermal signals are realized on the studied sample, providing fruitful informations from within the silica gel. These $2 \mathrm{D}$ and $3 \mathrm{D}$ imaging techniques could find many applications as new tools for nondestructive control problematics, ranging from material design to living tissue inspection.
\end{abstract}

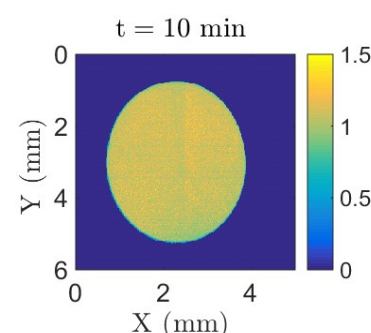

$\mathrm{X}(\mathrm{mm})$

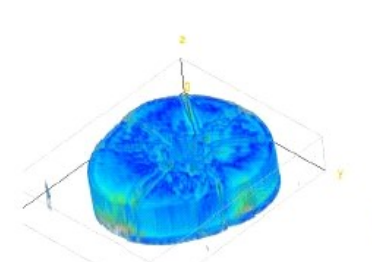

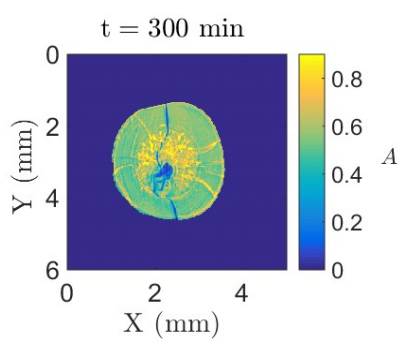

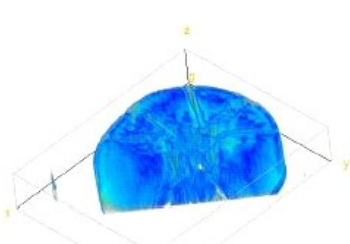

$\mathrm{t}=200 \mathrm{~min}$
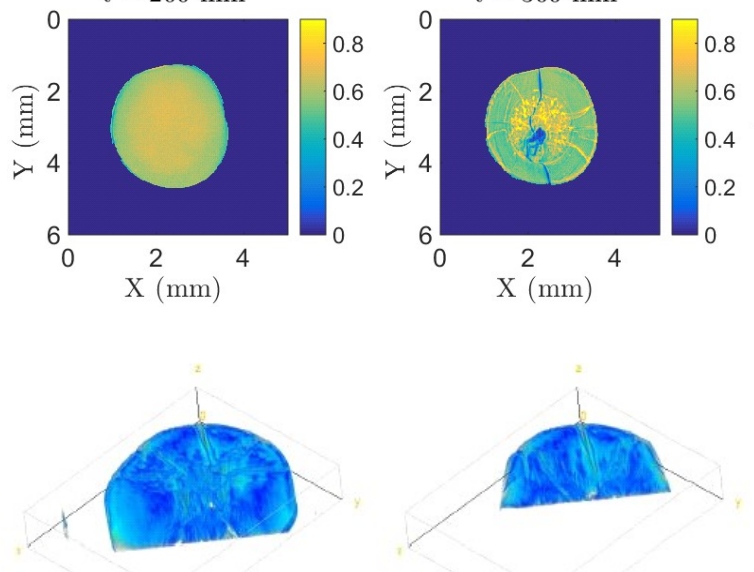

Fig. 1. (Top) Images of absorbance $(\lambda=4 \mu \mathrm{m})$ of the silica dispersion drop at 3 different instants of its drying.

(Bottom) Tomographs of transmitted intensity $(\lambda=4 \mu \mathrm{m})$ of the ending result of a confined drying

\section{REFERENCES}

[1] M. Romano, M. Ryu, J. Morikawa, J. Batsale, and C. Pradere, "Simultaneous microscopic measurements of thermal and spectroscopic fields of a phase change material, Infrared Physics \& Technology (2016)

[2] C. Loussert, A. Bouchaudy, and J.-B. Salmon, "Drying dynamics of a charged colloidal dispersion in a confined drop," Physical Review Fluids, Dec. 2016.

[3] C. Pradere, C. Hany, J. Toutain, and J.-C. Batsale, "Thermal Analysis for Velocity, Kinetics, and Enthalpy Reaction Measurements in Microfluidic Devices," Experimental Heat Transfer (2009) 